\title{
POISSON GROUPS AND DIFFERENTIAL GALOIS THEORY OF SCHROEDINGER EQUATION ON THE CIRCLE
}

\author{
IAN MARSHALL AND MICHAEL SEMENOV-TIAN-SHANSKY
}

\begin{abstract}
We combine the projective geometry approach to Schroedinger equations on the circle and differential Galois theory with the theory of Poisson Lie groups to construct a natural Poisson structure on the space of wave functions (at the zero energy level). Applications to KdV-like nonlinear equations are discussed. The same approach is applied to $2^{\text {nd }}$ order difference operators on a one-dimensional lattice, yielding an extension of the lattice Poisson Virasoro algebra.
\end{abstract}

\section{INTRODUCTION}

It is well known that the space $\mathcal{H}$ of Schroedinger operators on the circle

$$
H=-\partial_{x}^{2}-u, \quad u \in C^{\infty}\left(S^{1}\right), \quad S^{1} \simeq \mathbb{R} / 2 \pi \mathbb{Z},
$$

may be regarded as the phase space for the KdV hierarchy (with periodic boundary conditions). It carries a family of natural Poisson structures which play an important rôle in the Hamiltonian description of the KdV flows. In this letter we shall be concerned with the so called second Poisson structure for the KdV equation associated with the third order differential operator

$$
l=\frac{1}{2} \partial_{x}^{3}+u \partial_{x}+\partial_{x} u .
$$

This Poisson structure may be regarded as the Lie-Poisson bracket associated with the Virasoro algebra and arises as a result of the identification of $\mathcal{H}$ with (a hyperplane in) the dual space of the Virasoro algebra. Our aim is to describe its extension to the space of wave functions, i.e., of solutions of the Schroedinger equations (at zero energy level). Despite its apparent simplicity, this question involves several nontrivial points and has not been fully explored in the existing literature 1

According to elementary theory, for a given $u$ the space $V=V_{u}$ of solutions of the Schroedinger equation

$$
-\psi^{\prime \prime}-u \psi=0
$$

is 2-dimensional and for any two solutions $\phi, \psi$ their wronskian $W=\phi \psi^{\prime}-\phi^{\prime} \psi$ is constant. An element $w \in V$ may be regarded as a non-degenerate quasi-periodic plane curve (the non-degeneracy condition means that $w \wedge w^{\prime}$ is nowhere zero). There

\footnotetext{
${ }^{1}$ We do not discuss the generalization to the case of higher order differential operators, as well as relation to the Drinfeld-Sokolov theory [DS1]. These questions will be addressed in a separate publication.
} 
exists a matrix $M \in S L(2)$ (the monodromy matrix) such that, writing elements of $V$ as row vectors $w=(\phi, \psi)$,

$$
w(x+2 \pi n)=w(x) M^{n}, \quad n \in \mathbb{Z} .
$$

The group $G=S L(2)$ acts naturally on $V$ (preserving the wronskian) by right multiplication. $G$ plays a key rôle in the geometry of $\mathcal{H}$ in its double guise of the differential Galois group of equation (2) and of the group of projective transformations. Both aspects are completely classical; the novel element introduced in the present paper consists in their interaction with the Poisson geometry.

Let us recall how the Schroedinger equation is seen from the viewpoint of projective geometry. The following assertion is well known (see [OT]).

Theorem. (i) Any pair of linearly independent solutions of the Schroedinger equation defines a non-degenerate quasi-periodic projective curve $\gamma: \mathbb{R} \rightarrow \mathbb{C} P_{1}$ such that $\gamma(x+2 \pi)=\gamma(x) M$. Any two projective curves associated with a given Schroedinger equation are related by a global projective transformation. (ii) Conversely, any nondegenerate quasi-periodic projective curve may be lifted to a non-degenerate curve in $\mathbb{C}^{2}$ such that its wronskian is equal to 1.

In more abstract language, $\mathcal{H}$ is the space of projective connections on the circle. For a given $H_{u}=-\partial^{2}-u \in \mathcal{H}$ there is a natural projective line bundle $\mathcal{P}_{u} \rightarrow$ $S^{1}$; the quasi-periodic projective curve referred to above is its covariantly constant section, and the group $G=S L(2)$, or, more precisely, the associated projective group $P S L(2)=S L(2) /\{ \pm 1\})$, its structure group.

Without restricting the generality we may fix an affine coordinate on $\mathbb{C} P_{1}$ in such a way that $\infty$ corresponds to the zeros of the second coordinate $\psi$ of the point on the plane curve; with this choice $\gamma$ is replaced with the affine curve $x \mapsto \eta(x)=$ $\phi(x) / \psi(x)$. The potential $u$ may be restored from $\eta$ by the formula

$$
u=\frac{1}{2} S(\eta)
$$

where $S$ is the Schwarzian derivative

$$
S(\eta)=\frac{\eta^{\prime \prime \prime}}{\eta^{\prime}}-\frac{3}{2}\left(\frac{\eta^{\prime \prime}}{\eta^{\prime}}\right)^{2},
$$

which has the crucial property of being invariant under projective transformations

$$
\eta \mapsto \frac{a \eta+c}{b \eta+d}
$$

induced by the right action of $G$.

The space $\mathcal{V}$ of all quasi-periodic plane curves with wronskian 1 , or the equivalent space of projective curves (together with the associated monodromy matrices) encodes all information about Schroedinger operators. In W1 G. Wilson considered the extension of the KdV hierarchy to this space. To put it in a more formal way let us note that the natural "algebra of observables" associated with the KdV equation 
consists of local functionals of the form

$$
F[u]=\int_{0}^{2 \pi} F\left(u, \partial_{x} u, \partial_{x}^{2} u, \ldots\right) d x,
$$

where $F$ is a polynomial (or, more generally, a rational) function of $u$ and of its derivatives. We can identify the observable $F[u]$ and the corresponding density; in other words, our basic algebra of observables is identified with the differential field $\mathbb{C}\langle u\rangle$. In the same way, we can associate with the space of solutions of the Schroedinger equation a bigger differential field $\mathbb{C}\langle\phi, \psi\rangle$. Clearly, $\mathbb{C}\langle\phi, \psi\rangle \supset \mathbb{C}\langle u\rangle$; as a matter of fact, $\mathbb{C}\langle u\rangle$ is isomorphic to the differential subfield of $G$-invariants and hence $\mathbb{C}\langle\phi, \psi\rangle \supset \mathbb{C}\langle u\rangle$ is a differential Galois extension with differential Galois group $G=S L(2)$ (we shall speak below simply of Galois groups and Galois extensions, for short). Various subgroups of $G$ give rise to intermediate differential fields. In particular, for $Z=\{ \pm I\}$ the associated subfield of invariants is naturally isomorphic to $\mathbb{C}\langle\eta\rangle$; since $Z$ is the center of $G$, the extension $\mathbb{C}\langle\eta\rangle \supset \mathbb{C}\langle u\rangle$ is again a Galois extension with the Galois group $P S L(2)=S L(2) / Z 2$

Let $B$ the subgroup of lower triangular matrices; its field of invariants $\mathbb{C}\langle\phi, \psi\rangle^{B}$ may be identified with $\mathbb{C}\langle v\rangle$, where $v=\frac{1}{2} \frac{\eta^{\prime \prime}}{\eta^{\prime}}$. One has $u=v^{\prime}-v^{2}$, which is the classical Miura transform. Note that since $B$ is not normal in $G, \mathbb{C}\langle v\rangle \supset \mathbb{C}\langle u\rangle$ is not a Galois extension, and hence, as noted by Wilson W1, the treatment of the Miura transform requires the introduction of the 'universal covering' algebra $\mathbb{C}\langle\phi, \psi\rangle$.

The natural idea explored in [W1] is the possibility to lift the KdV flows originally defined on $\mathcal{H}$ to the bigger space $\mathcal{V}$. An important ingredient of such an extension is to equip $\mathcal{V}$ with a Poisson structure or its substitute. Wilson's point of view is to look at the symplectic form, because it may be naturally pulled back (at the expense of becoming degenerate, see [W1]). A closer look at the situation reveals yet another difficulty: the relevant 'variational' 2-form is an integral of a density whose differential is not identically zero; rather it is a closed form on the circle and hence its contribution disappears only if we may discard 'total derivatives'. This convention, adopted in formal variational calculus, greatly simplifies many formulae, but sometimes hides important "obstruction terms". In Wilson's paper this difficulty is avoided by the tacit assumption that the monodromy matrix is equal to 1. Without this assumption the degenerate 2-forms discussed in his paper are not closed; hence finally his approach is intrinsically close to the quasi-Hamiltonian formalism of Alekseev, Malkin and Meinrenken [AMM]. An alternative approach, followed in the present paper, is to look at the Poisson structure. Of course, Poisson brackets cannot be pulled back, and hence we have to guess a Poisson structure on the extended algebra and then check its consistency with the original bracket. Our strategy is based on the projective point of view outlined above. Although the space of projective curves is our main object, it is natural to start with the much bigger

\footnotetext{
${ }^{2}$ The Galois theory point of view was implicit in the old paper of Drinfeld and Sokolov [DS2], where wave functions for different values of energy are considered, leading to to an extended class of "equations of KdV type". Generically, the associated Galois group becomes in this case the product of several copies of $S L(2)$.
} 
space $\mathcal{W}$ of all quasi-periodic plane curves,

$$
\mathcal{W}=\{(w=(\phi, \psi), M) \mid w(x+2 \pi)=w(x) M\} .
$$

The space $\mathcal{W}$ contains the set $\mathcal{W}^{\prime}$ of all non-degenerate plane curves with non-zero wronskian as an open subset. Let $\mathcal{C}:=C^{\infty}\left(\mathbb{R} / 2 \pi \mathbb{Z}, \mathbb{C}^{\times}\right)$be the scaling group which acts on $\mathcal{W}$ via

$$
f \cdot(w, M)=(f w, M) .
$$

Clearly, $\mathcal{C}$ acts freely on $\mathcal{W}^{\prime}$ and the quotient may be identified with $\mathcal{V}$. The action of the linear group $G=S L(2)$ on $\mathcal{W}$ is via $g: w \mapsto w \cdot g, M \mapsto g^{-1} M g$. The key condition which we use to restrict the choice of the Poisson structure on $\mathcal{W}$ is its covariance with respect to the group action. This condition puts us in the framework of Poisson group theory, as it allows both $\mathcal{C}$ and $G$ to carry nontrivial Poisson structures, although it does not presume any a priori choice of these structures. As it happens, the covariance condition together with the natural constraint on the wronskian make their choice almost completely canonical. (In particular, the Poisson bracket on $G$ is fixed up to scaling and conjugation; it is of the standard "quastriangular" type and the case of zero bracket is excluded.) Let us note that the Poisson structure on $\mathcal{W}$ constructed in this way is closely related to the so called exchange algebras discovered in the end of 1980s [B1]. The point of view adopted in the present paper provides a useful and nontrivial complement to these old results in making explicit the hidden Poisson group aspects of differential Galois theory. It provides a natural route to the usual Virasoro algebra and also to its discrete analogue as discussed in [FT], [V], [B2] and [FRS].

\section{A REview of Poisson Lie groups}

Let $G$ be a Lie group with Lie algebra $\mathfrak{g}$. A Poisson structure on $G$ is called multiplicative if the multiplication

$$
m: G \times G \rightarrow G
$$

is a Poisson mapping. A Lie group equipped with a multiplicative Poisson bracket is called a Poisson Lie group.

Any multiplicative Poisson bracket on $G$ identically vanishes at its unit element $e \in G$; its linearization at $e$ gives rise to the structure of a Lie algebra on the dual space $\mathfrak{g}^{*}$; multiplicativity then implies that the dual of the commutator map $[]:, \mathfrak{g}^{*} \times \mathfrak{g}^{*} \rightarrow \mathfrak{g}^{*}$ is a 1 -cocycle on $\mathfrak{g}$. A pair $\left(\mathfrak{g}, \mathfrak{g}^{*}\right)$ with these properties is called a Lie bialgebra. A fundamental theorem, due to Drinfeld, asserts that a multiplicative Poisson bracket on $G$ is completely determined by its linearization and hence there is an equivalence between the category of Poisson Lie groups (whose morphisms are Lie group homomorphisms which are also Poisson mappings) and the category of Lie bialgebras (whose morphisms are homomorphisms of Lie algebras such that their duals are homomorphisms of the dual algebras).

An action $G \times \mathcal{M} \rightarrow \mathcal{M}$ of a Poisson group on a Poisson manifold $\mathcal{M}$ is called a Poisson action if this mapping is Poisson; in other words, for $F, H \in \operatorname{Fun}(\mathcal{M})$, their 
Poisson bracket at the transformed point $g \cdot m \in \mathcal{M}$ may be computed as follows:

$$
\{F, H\}_{\mathcal{M}}(g \cdot m)=\{\hat{F}(m, \cdot), \hat{H}(m, \cdot)\}_{G}(g)+\{\hat{F}(\cdot, g), \hat{H}(\cdot, g)\}_{\mathcal{M}}(m),
$$

where in the r.h.s. we set $\hat{F}(m, g)=F(g \cdot m), \hat{H}(m, g)=H(g \cdot m)$ and treat them as functions of two variables $g \in G, m \in \mathcal{M}$. In that case we shall also say that the Poisson bracket on $\mathcal{M}$ is $G$-covariant. The choice of the basic ring of functions on $\mathcal{M}$ depends on the context; we may work, for instance, in the $C^{\infty}$ setting or, alternatively, consider the rings of polynomial or rational functions on the appropriate manifolds.

It is sometimes useful to restrict the action $G \times \mathcal{M} \rightarrow \mathcal{M}$ to a subgroup of $G$. A natural class of subgroups of $G$ are those Lie subgroups which are also Poisson submanifolds for which the inherited Poisson structure is of course multiplicative. This class, however, is too restricted, since a Poisson Lie group may have very few Poisson subgroups and a wider class consists of the so called admissible subgroups. A subgroup $H \subset G$ of a Poisson Lie group $G$ is called admissible if the subalgebra of $H$-invariants $\operatorname{Fun}(\mathcal{M})^{H} \subset \operatorname{Fun}(\mathcal{M})$ is closed with respect to the Poisson bracket. A simple admissibility criterion is stated as follows. Let $\mathfrak{h} \subset \mathfrak{g}$ be the Lie algebra of $H$ and $\mathfrak{h}^{\perp} \subset \mathfrak{g}^{*}$ its annihilator in $\mathfrak{g}^{*}$. Then $H \subset G$ is admissible if and only if $\mathfrak{h}^{\perp} \subset \mathfrak{g}^{*}$ is a Lie subalgebra; $H \subset G$ is a Poisson subgroup if and only if $\mathfrak{h}^{\perp}$ is an ideal in $\mathfrak{g}^{*}$.

Let us assume that $H$ is admissible and that the quotient space $\mathcal{M} / H$ is smooth, so that case we may identify $\operatorname{Fun}(\mathcal{M} / H)$ with $\operatorname{Fun}(\mathcal{M})^{H}$ and hence the quotient space inherits the Poisson structure. This is the basis of Poisson reduction, originally introduced by Lie.

The only nontrivial example which we need in the present paper is the projective group $G=S L(2)$ (or $P S L(2)$ ). The group $G=S L(2, \mathbb{C})$ carries a family of natural Poisson structures called the Sklyanin brackets which make it a Poisson Lie group. These Poisson structures are parameterized by the choice of a classical r-matrix $r \in \mathfrak{g} \wedge \mathfrak{g}$; for $\mathfrak{g}=\mathfrak{s l}(2)$ the classical Yang-Baxter equation does not impose any restrictions on the choice of $r$, so any element of $\mathfrak{g} \wedge \mathfrak{g}$ gives rise to a Poisson bracket on $G$. It is specified by the set of Poisson bracket relations for the matrix coefficients of $G$ (regarded as generators of its affine ring). In usual tensor notation we have

$$
\left\{g_{1}, g_{2}\right\}=\left[r, g_{1} g_{2}\right] \text {, }
$$

where in the r.h.s. we regard $r \in \mathfrak{g} \wedge \mathfrak{g}$ and $g_{1} g_{2}=g \otimes g$ as elements of $\operatorname{Mat}(2) \otimes$ $\operatorname{Mat}(2) \simeq \operatorname{Mat}(4)$ and compute the commutator in Mat(4).

Let $h, e, f$ be the standard generators of $\mathfrak{s l}(2)$. Up to the natural equivalence there exist three types of classical r-matrices:

(a) $r=0$;

(b) $r=h \wedge f$

(c) $r=\epsilon e \wedge f$, where $\epsilon$ is a scaling parameter.

They correspond to three types of $G$-orbits in $\mathfrak{g}$. Case (a) gives trivial bracket; case (c) is generic; case (b) (the so called triangular r-matrix) is degenerate. The standard 
Poisson bracket on $G$ which corresponds to case (c) is given by the following set of relations for the matrix coefficients of $g=\left(\begin{array}{ll}\alpha & \beta \\ \gamma & \delta\end{array}\right)$, we have

$$
\begin{array}{ll}
\{\alpha, \beta\}=\epsilon \alpha \beta, & \{\alpha, \gamma\}=\epsilon \alpha \gamma \\
\{\beta, \delta\}=\epsilon \beta \delta, & \{\gamma, \delta\}=\epsilon \gamma \delta \\
\{\beta, \gamma\}=0, \quad\{\alpha, \delta\}=2 \epsilon \beta \gamma
\end{array}
$$

Notice that $\operatorname{det} g=\alpha \delta-\beta \gamma$ is a Casimir function and hence the Poisson bracket is well defined on the coordinate ring of $S L(2)$ and even of $P S L(2)$.)

In the sequel we shall be mainly concerned with the standard bracket (6) $)$. We shall see that the covariance condition together with the wronskian constraint fix the Poisson structure on $G$ uniquely up to scaling and conjugation; in particular, r-matrices of types (a) and (b) are excluded. It will be important for us to have an explicit description of the dual Poisson group associated with the standard r-matrix (of type (c)) on $\mathfrak{g}$.

Let $\mathfrak{b}_{ \pm} \subset \mathfrak{g}$ be the opposite Borel subalgebras of $\mathfrak{g}=\mathfrak{s l}(2)$ which consist of upper (respectively, lower) triangular matrices. The dual Lie algebra $\mathfrak{g}^{*}$ associated with the standard r-matrix may be identified with the subalgebra of $\mathfrak{b}_{+} \oplus \mathfrak{b}_{-}$,

$$
\mathfrak{g}^{*}=\left\{\left(X_{+}, X_{-}\right) \in \mathfrak{b}_{+} \oplus \mathfrak{b}_{-} \mid \operatorname{diag} X_{+}+\operatorname{diag} X_{-}=0\right\} .
$$

We conclude, in particular, that the standard Cartan subgroup $H$, Borel subgroups $B_{ \pm}$and unipotent subgroups $N \pm \subset B_{ \pm}$are admissible subgroups of $G$. (Of course, this is not true for conjugate subgroups!)

The Lie group $G^{*}$ associated with $\mathfrak{g}^{*}$ may be identified with the subgroup in $B_{+} \times B_{-}$,

$$
G^{*}=\left\{\left(b_{+}, b_{-}\right) \in B_{+} \times B_{-} \mid \operatorname{diag} b_{+} \cdot \operatorname{diag} b_{-}=I\right\} .
$$

It carries a natural Poisson bracket which makes it a Poisson Lie group; this is the dual Poisson Lie group of $G$. The mapping

$$
G^{*} \rightarrow G:\left(b_{+}, b_{-}\right) \mapsto M=b_{+} b_{-}^{-1}
$$

maps $G^{*}$ onto an open dense subset in $G$; the induced Poisson structure on extends smoothly to the entire manifold $G$. Explicitly it is described by the following formula:

$$
\left\{M_{1}, M_{2}\right\}=M_{1} M_{2} r+r M_{1} M_{2}-M_{2} r_{+} M_{1}-M_{1} r_{-} M_{2},
$$

where $r_{ \pm}=r \pm \epsilon t$ and $t \in \mathfrak{g} \otimes \mathfrak{g}$ stands for the tensor Casimir element. This Poisson structure on $G$ has a number of remarkable properties; in particular, its symplectic leaves are conjugacy classes in $G$; moreover, this bracket is covariant with respect to the action of $G$ (equipped with the bracket (6) ) by conjugation. Conversely, the only Poisson structure on $G$ (now regarded as a $G$-space, not as a group) which is Poisson covariant with respect to the action of $G$ by conjugation is that given by (8). 


\section{The space of Wave functions as a Poisson space}

We shall assume in the sequel that all functions take values in $\mathbb{C}$. For $M \in$ $S L(2, \mathbb{C})$ let $\mathcal{W}_{M}$ be the space of smooth quasi-periodic plane curves,

$$
\mathcal{W}_{M}=\left\{w: \mathbb{R} \rightarrow \mathbb{C}^{2} \mid w(x+2 \pi)=w(x) M \text { for all } x\right\},
$$

where $w$ is denoted by a row vector. Let $\mathcal{W}$ be the set of pairs,

$$
\mathcal{W}=\left\{(w, M) \mid M \in S L(2, \mathbb{C}), w \in \mathcal{W}_{M}\right\}
$$

The wronskian $W: \mathcal{W} \rightarrow \mathbb{C}$ is defined by the standard formula

$$
W(\phi, \psi)=\phi \psi^{\prime}-\phi^{\prime} \psi
$$

and we define $\mathcal{W}^{\prime} \subset \mathcal{W}$ to be the open subset consisting of non-degenerate curves, i.e. having non-zero wronskian.

We want to find the most general Poisson structure on $\mathcal{W}$ which is covariant with respect to the right action of $G=S L(2, \mathbb{C})$ and to the action of the scaling group $\mathcal{C}$. This structure appears to be partially rigid. It is convenient to describe this Poisson structure by giving the Poisson brackets of the 'evaluation functionals' which assign to wave functions $\phi, \psi$ their values at the running point $x \in \mathbb{R}$. The covariance with respect to the local scaling group implies that these brackets are quadratic and local, i.e., depend only on the values of $\phi, \psi$ at the given points.

Lemma 2.1. Assume that the Poisson bracket on $\mathcal{W}$ is covariant with respect to the action of $\mathcal{C}$. Then the Poisson structure on $\mathcal{C}$ is trivial and, writing $w=(\phi, \psi)$, the bracket of evaluation functionals has the form

$$
\begin{aligned}
& \{\phi(x), \phi(y)\}=A(x, y) \phi(x) \phi(y), \quad\{\psi(x), \psi(y)\}=D(x, y) \psi(x) \psi(y), \\
& \{\phi(x), \psi(y)\}=B(x, y) \phi(x) \psi(y)+C(x, y) \phi(y) \psi(x) .
\end{aligned}
$$

It is natural to assume that the bracket (11) is translation invariant, i.e., the structure functions depend only on the difference $x-y$. Using tensor notation, we can write these Poisson brackets in the following condensed form:

$$
\left\{w_{1}(x), w_{2}(y)\right\}=w_{1}(x) w_{2}(y) R(x, y),
$$

where $w(x)=(\phi(x), \psi(x))$ and we write the tensor product $w_{1}(x) w_{2}(y)$ as a row vector of length 4 ; the matrix $R(x, y) \in \operatorname{Mat}(4)$ is given by

$$
R(x, y)=\left(\begin{array}{cccc}
A(x-y) & 0 & 0 & 0 \\
0 & B(x-y) & -C(y-x) & 0 \\
0 & C(x-y) & -B(y-x) & 0 \\
0 & 0 & 0 & D(x-y)
\end{array}\right)
$$

Poisson brackets of this type were first studied in [B1] (for a special choice of $R$ ).

It is convenient to drop temporarily the Jacobi identity condition and to consider all (generalized) Poisson brackets which are covariant with respect to the Galois group action. 
Lemma 2.2. Let us assume that the Poisson bracket (12) is right-G-invariant; then the exchange matrix has the structure

$$
R_{0}(x, y)=a(x-y) I+\left(\begin{array}{cccc}
0 & 0 & 0 & 0 \\
0 & c(x-y) & -c(x-y) & 0 \\
0 & c(x-y) & -c(x-y) & 0 \\
0 & 0 & 0 & 0
\end{array}\right)
$$

where $a$ and $c$ are arbitrary odd functions.

Lemma 2.3. Fix an arbitrary $r$-matrix $r \in \mathfrak{g} \wedge \mathfrak{g}$ and equip $G$ with the corresponding Sklyanin bracket (5). Let us assume that the Poisson bracket (12) is right-Gcovariant; then the exchange matrix has the structure

$$
R_{r}(x, y)=R_{0}(x, y)+r
$$

where we write $r \in \mathfrak{g} \wedge \mathfrak{g} \subset \operatorname{Mat}(2) \otimes \operatorname{Mat}(2)$ as a $4 \times 4$-matrix in the standard way.

For $\mathfrak{g}=\mathfrak{s l}(2)$ the classical Yang-Baxter equation does not impose any restrictions on the choice of $r$; indeed, it amounts to the requirement that the Schouten bracket $[r, r] \in \mathfrak{g} \wedge \mathfrak{g} \wedge \mathfrak{g}$ should be ad $\mathfrak{g}$-invariant, but for $\mathfrak{g}=\mathfrak{s l}(2)$ we have $\wedge^{3} \mathfrak{g} \simeq \mathbb{C}$. Still, we must distinguish two cases:

- $[r, r]=0$, which happens when $r=0$ or $r$ is triangular (cases (a) and (b) of the classification in section 2 above).

$-[r, r]=-\epsilon^{2} \neq 0$, which happens when $r$ is quasitriangular (case (c)).

Since $R_{r}$ in (14) is the sum of 2 terms, the Schouten bracket $[r, r]$ gives an extra term to the Jacobi identity for the corresponding exchange bracket.

Lemma 2.4. The exchange bracket (12) with exchange matrix (14) satisfies the Jacobi identity if and only if

$$
c(x-y) c(y-z)+c(y-z) c(z-x)+c(z-x) c(x-y)=0
$$

in cases (a) and (b) and

$$
c(x-y) c(y-z)+c(y-z) c(z-x)+c(z-x) c(x-y)=-\epsilon^{2}
$$

in case (c).

Functional equation (16) is a version of the so called Rota-Baxter equation. To solve it, one can put $c(x)=\epsilon C(x)$ and express $C$ as a Cayley transform,

$$
C(x)=\frac{f(x)+1}{f(x)-1}
$$

then (16) immediately yields for $f$ the standard 2-cocycle relation

$$
f(x-y) f(y-z) f(z-x)=1 .
$$

The obvious solution is thus $C_{\lambda}(x-y)=\operatorname{coth} \lambda(x-y)$, where $\lambda$ is a parameter. Setting $\lambda \rightarrow \infty$, we obtain a particular solution $C(x-y)=\operatorname{sign}(x-y)$. We shall see that this special solution is the only one which is compatible with the constraint $W=1$. The solution of the degenerate equation (15) is $c(x)=1 / x$. 
So far, the most general Poisson structure on $\mathcal{W}$ still contains functional moduli and a free parameter. As is easy to check, the Poisson brackets for the ratio $\eta=\phi / \psi$ do not depend on $a$ :

Proposition 2.5. We have

$$
\{\eta(x), \eta(y)\}=\epsilon\left(\eta(x)^{2}-\eta(y)^{2}\right)-c(x-y)(\eta(x)-\eta(y))^{2} .
$$

Remark 2.6. Formula (17) defines a family of $G$-covariant Poisson brackets on the space of projective curves. However, in order to establish a connection between these brackets and Schroedinger operators we must take into account the wronskian constraint which restricts the choice of $c$. The second structure function $a$ drops out after projectivization and is not restricted by the Jacobi identity. We shall see, however, that the wronskian constraint suggests a natural way to choose $a$ as well. An interpretation of the general family (17) of Poisson brackets remains an open question.

Our next proposition describes the basic Poisson bracket relations for the wronskian:

Proposition 2.7. We have

$$
\begin{aligned}
\{W(x), \phi(y)\}=(c(x-y)-2 a(x, y)) & W(x) \phi(y) \\
& -c^{\prime}(x-y) \phi(x)[\phi(x) \psi(y)-\psi(x) \phi(y)] .
\end{aligned}
$$

By symmetry, a similar formula holds for $\{W(x), \psi(y)\}$.

Formula (18) immediately leads to the following crucial observation:

Proposition 2.8. The constraint $W=1$ is compatible with the Poisson brackets for scaling invariant $\eta$ if and only if the last term in (18) is identically zero; this is possible if and only if $C^{\prime}(x-y)$ is a multiple of $\delta(x-y)$, i.e., if $C(x-y)$ is a multiple of $\operatorname{sign}(x-y)$.

It is important that the wronskian constraint excludes the possibility that $\epsilon=0$ and hence the corresponding Poisson structure on $G$ is conjugate to the standard one (case (c)). From now on, without restricting the generality, we fix $\epsilon=1$.

Proposition 2.9. Let us assume that $c(x-y)=\operatorname{sign}(x-y)$; then the Poisson bracket relations for the wronskian are given by:

$$
\{W(x), W(y)\}=(\operatorname{sign}(x-y)-2 a(x, y)) W(x) W(y),
$$

or, equivalently

$$
\{\log W(x), \log W(y)\}=(\operatorname{sign}(x-y)-2 a(x, y)) .
$$

Formulae (18) and (19) suggest the following distinguished choice of $a$ :

Proposition 2.10. Assume that a is so chosen that

$$
\operatorname{sign}(x-y)-2 a(x, y)=\delta^{\prime}(x-y) .
$$


(In other words, $a(x, y)$ is the distribution kernel of the operator $\frac{1}{2}\left(\partial^{-1}-\partial\right)$.) Then: (i) The logarithms of wronskians form a Heisenberg Lie algebra, the central extension of the abelian Lie algebra of $\mathcal{C}$. (ii) Let $\mathcal{C}^{\prime}=\mathcal{C} / \mathbb{C}^{*}$ be the quotient of the scaling group over the subgroup of constants; $\log W$ is the moment map for the action of $\mathcal{C}^{\prime}$ on $\mathcal{W}$.

Recall that according to the general theory the Poisson bracket relations for the moment map may reproduce the commutation relations for a central extension of the original Lie algebra. This is precisely what happens in the present case.

With this choice of $a$ and $C$ the Poisson geometry of the space $\mathcal{V}$ of wave functions becomes finally quite transparent: $\mathcal{V}$ arises as a result of Hamiltonian reduction with respect to $\mathcal{C}$ over the zero level of the associated moment map. The constraint set $\log W=0$ is (almost) non-degenerate (i.e., this is a $2^{\text {nd }}$ class constraint, according to Dirac). The projective invariants commute with the wronskian and hence their Poisson brackets are not affected by the constraint 3

The description of the Poisson structure on $\mathcal{V}$ is completed by the Poisson brackets for the monodromy.

Proposition 2.11. The Poisson covariant brackets for the monodromy have the form

$$
\begin{aligned}
\left\{w(x)_{1}, M_{2}\right\} & =w(x)_{1}\left[M_{2} r_{+}-r_{-} M_{2}\right] \\
\left\{M_{1}, M_{2}\right\} & =M_{1} M_{2} r+r M_{1} M_{2}-M_{2} r_{+} M_{1}-M_{1} r_{-} M_{2} .
\end{aligned}
$$

The Poisson bracket for the monodromy is precisely the Poisson bracket of the dual group $G^{*}$ described in (8) . In other words, the 'forgetting map' $\mu:(w, M) \mapsto M$ is a Poisson morphism from $\mathcal{W}$ into the dual group $\left.G^{*}\right]$ This mapping is of special importance.

Proposition 2.12. The mapping $\mu$ is the non-abelian moment map5 associated with the right action of $G$ on $\mathcal{W}$.

Let us now list the Poisson bracket relations in the differential algebra $\mathbb{C}\langle\eta\rangle$ and its various subalgebras which correspond to different admissible subgroups of $G$.

\footnotetext{
${ }^{3}$ With this choice of $a$ the Poisson structure on $\mathcal{W}$ becomes non-degenerate; for other possible choices this may be not true. For example, the opposite possibility is to set $\operatorname{sign}(x-y)-2 a(x, y)=$ 0 . This makes the bracket on $\mathcal{W}$ highly degenerate; its kernel is eliminated by the wronskian constraint, and the reduced structure remains the same. While logically possible, the resulting picture is much less attractive.

${ }^{4}$ The Poisson bracket (8) is ubiquitous in various problems related to monodromy; another striking example, which is very close to our present context, is its rôle in the theory of isomonodromic deformations described in the very interesting paper of P.Boalch [Bo].

${ }^{5}$ We refer the reader for instance to $[\mathrm{BB}$. for the general definition of non-abelian moment maps associated with Poisson group actions.
} 
Proposition 2.13. (i) Consider the tower of differential extensions

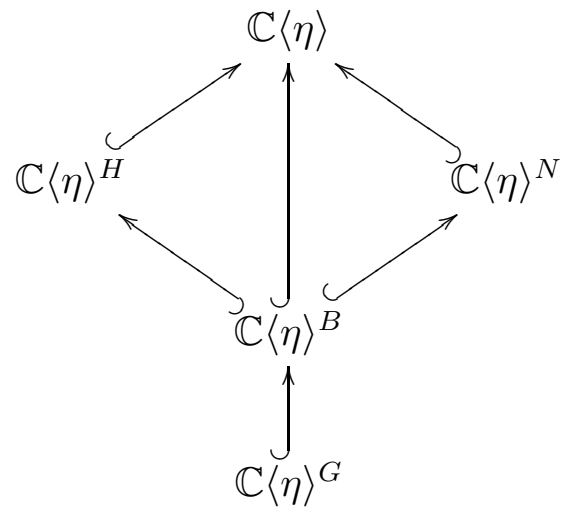

All arrows in this commutative diagram are Poisson morphisms.

(ii) The basic Poisson bracket relations in $\mathbb{C}\langle\eta\rangle$ are given by

$$
\{\eta(x), \eta(y)\}=\eta(x)^{2}-\eta(y)^{2}-\operatorname{sign}(x-y)(\eta(x)-\eta(y))^{2} .
$$

(iii) We have $\mathbb{C}\langle\eta\rangle^{N} \simeq \mathbb{C}\langle\theta\rangle$, where $\theta:=\eta^{\prime}$; moreover,

$$
\{\theta(x), \theta(y)\}=2 \operatorname{sign}(x-y) \theta(x) \theta(y) .
$$

(iv) The subalgebra of $B$-invariants is generated by $v:=\frac{1}{2} \eta^{\prime \prime} / \eta^{\prime}=\frac{1}{2} \theta^{\prime} / \theta$; we have:

$$
\{v(x), v(y)\}=\frac{1}{2} \delta^{\prime}(x-y) .
$$

(v) The subalgebra of $G$-invariants is generated by $u=\frac{1}{2} S(\eta)=v^{\prime}-v^{2}$; we have:

$$
\{u(x), u(y)\}=\frac{1}{2} \delta^{\prime \prime \prime}(x-y)+\delta^{\prime}(x-y)[u(x)+u(y)] .
$$

Formula (25) reproduces the standard Virasoro algebra; in other words, the Poisson algebra (22) constructed from general covariance principles is indeed an extension of the Poisson-Virasoro algebra.

Remark 2.14. The Poisson bracket relations (23) - (25) listed above are particularly simple, since their r.h.s. is algebraic. Because the basic Poisson bracket relations (22) are nonlocal, this need not always be the case. This is what happens in the case of $H$-invariants:

Proposition 2.15. (i) The differential subalgebra of $H$-invariants in $\mathbb{C}\langle\eta\rangle$ is generated by $\rho=\eta^{\prime} / \eta$. (ii) The Poisson brackets for $\rho$ have the form

$$
\{\rho(x), \rho(y)\}=2 \rho(x) \rho(y)\left[\sinh \int_{x}^{y} \rho(s) d s+\operatorname{sign}(x-y) \cosh \int_{x}^{y} \rho(s) d s\right] .
$$

It is well known that the standard KdV equation is generated with respect to the Virasoro bracket by the Hamiltonian

$$
H=\int u^{2} d x
$$


The Hamiltonians of all higher KdV equations are associated with trace identities for $H_{u}$ and hence are $G$-invariant; they generate a system of compatible commuting flows on all levels of the extension tower.

Proposition 2.16. The following commutative diagram which is formed by Poisson maps summarizes all information on the evolution equations generated by the standard Hamiltonian (26) and on the differential substitutions which relate these equations.

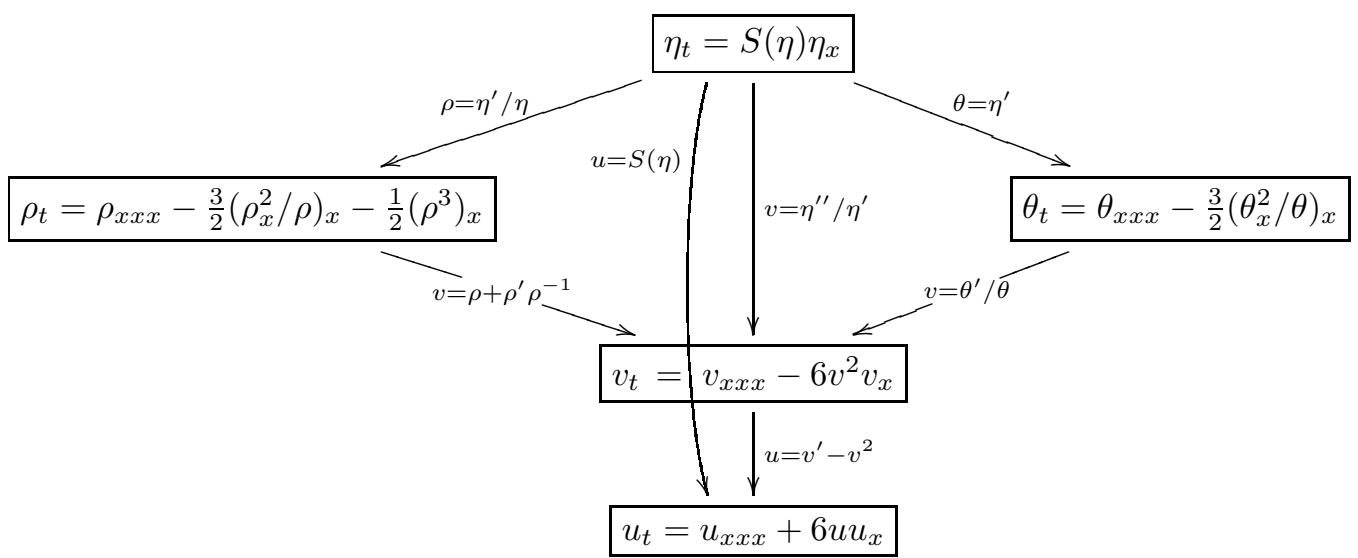

All equations in this diagram belong to the well known class of "equations of the KdV type". Their mutual relations were discussed by Wilson [W1], although the Hamiltonian description which we propose is totally different. Equation

$$
\eta_{t}=S(\eta) \eta_{x}=\eta_{x x x}-\frac{3}{2} \eta_{x x}^{2} / \eta_{x}
$$

is sometimes called the Schwarz-KdV equation; in [W1 George Wilson suggested for it the name "ur-KdV equation", due to its position atop the extension tower.

Remark 2.17. Equations which appear in the diagram form a rather small part in the general class of "equations of the KdV type" discussed in [SS], where a classification theorem is given for evolution equations of the form $u_{t}=u_{x x x}+F\left(u, u_{x}, u_{x x}\right)$ which admit nontrivial conservation laws. General equations of this type depend on several parameters and may include elliptic functions, as was first noticed by Calogero and Degasperis [CD. We expect that rational equations from this list will also fit into the Poisson group setting by bringing into play the wave functions for different values of energy, as suggested in [DS2].

\section{Discrete CASE}

The theory of the Schroedinger equation has a simple and natural lattice counterpart. Consider the $2^{\text {nd }}$ order difference equation on the one-dimensional lattice with periodic potential

$$
\phi_{n+2}+u_{n} \phi_{n+1}+\phi_{n}=0, \quad u_{n+N}=u_{n} .
$$


Let $\tau$ be the shift operator, $(\tau \phi)_{n}=\phi_{n+1}$. Equation (28) may be written in operator form as

$$
\left(\tau^{2}+u \tau+1\right) \phi=0
$$

For a given $u$, the space of its solutions is two-dimensional; any two solutions $\phi, \psi$ have constant wronskian $W=\phi_{n} \psi_{n-1}-\phi_{n-1} \psi_{n}$. The monodromy matrix $M$ is defined in the standard way.

The projective description of discrete Schroedinger equations is given by the following theorem. To state it we need a few elementary notions. An ordered projective configuration is a map $\gamma: \mathbb{Z} \rightarrow \mathbb{C} P_{1}$; we shall simply speak of projective configurations, for short. A configuration is called non-degenerate if $\gamma_{n} \neq \gamma_{n+1}$. for all $n$. A plane configuration is a map $w: \mathbb{Z} \rightarrow \mathbb{C}^{2}$; it is called non-degenerate if $w_{n} \wedge w_{n+1} \neq 0$. We denote $w_{n}$ by the row vector $\left(\phi_{n}, \psi_{n}\right)$.

Theorem. (i) Any pair of linearly independent solutions of the discrete Schroedinger equation defines a non-degenerate quasi-periodic projective configuration $\gamma: \mathbb{Z} \rightarrow$ $\mathbb{C} P_{1}$ such that $\gamma_{n+N}=\gamma_{n} \cdot M$. Any two projective configurations associated with a given discrete Schroedinger equation are related by a global projective transformation.

(ii) Conversely, any non-degenerate quasi-periodic projective configuration may be lifted to a non-degenerate plane configuration such that its wronskian is equal to 1.

As before, we replace the projective line with its affine model putting $\eta_{n}=\phi_{n} / \psi_{n}$. The group $G=S L(2)$ is the (difference) Galois group of equation (28). Curiously, the potential $u$ itself is not a rational Galois invariant. A natural finite difference analog of the Schwarzian derivative is the cross-ratio,

$$
s_{n}[\eta]:=\left[\eta_{n}, \eta_{n+1}, \eta_{n+2}, \eta_{n+3}\right]=\frac{\eta_{n}-\eta_{n+2}}{\eta_{n}-\eta_{n+1}} \cdot \frac{\eta_{n+1}-\eta_{n+3}}{\eta_{n+2}-\eta_{n+3}}
$$

an elementary calculation yields

$$
s_{n}=u_{n} u_{n+1} .
$$

From now on we shall assume that the period $N$ of the lattice is odd. In this case the potential may be restored as the periodic solution of (30) (regarded as an equation for $u$ for given $\left.\left.s_{m}[\eta]\right|_{m=1} ^{N}\right)$; it belongs to a quadratic extension of $\mathbb{C}(\eta)^{G}=\mathbb{C}\left(u u^{\tau}\right) \subset$ $\mathbb{C}(u)$. Note that the resulting formula is non-local, that is, it depends on the values of $\eta_{m}$ for all $m$.

The Poisson structure on the space of discrete Schroedinger operators is much less obvious than in the continuous case; it may be regarded as a lattice analog of the Virasoro algebra. One version of its definition was proposed in [FRS] as a part of a more general theory, the q-difference version of the Drinfeld-Sokolov theory [DS1] which applies to q-difference equations of arbitrary order (see also [STSS]). Another definition of the lattice Virasoro algebra had been proposed earlier by Faddeev and Takhtajan [FT]. The projective point of view outlined in the present paper also yields a natural Poisson structure on the space of discrete Hill's operators; we shall see that it is identical to that introduced in [FRS] and is simply related to the Faddeev-Takhtajan bracket. 
In this section we shall denote by $\mathcal{W}$ the space of all plane quasi-periodic configurations and by $\mathcal{C}$ the discrete scaling group.

Proposition 3.1. (i) Let us assume that the Poisson structure on $\mathcal{W}$ is covariant with respect to the right action of $G$ and to the natural action of the scaling group. Then the bracket between the evaluation functionals is given by

$$
\left\{w_{m}^{1}(x), w_{n}^{2}(y)\right\}=w_{m}^{1}(x) w_{n}^{2}(y) R(m-n),
$$

where

$$
R(k)=R_{0}(k)+r, \quad R_{0}(k)=a_{k} I+\left(\begin{array}{cccc}
0 & 0 & 0 & 0 \\
0 & c_{k} & -c_{k} & 0 \\
0 & c_{k} & -c_{k} & 0 \\
0 & 0 & 0 & 0
\end{array}\right),
$$

(we omitted Poisson bracket relations for the monodromy which remain the same as before). Here $a_{k}$ is an arbitrary odd function and $c_{k}$ is an odd function which satisfies

$$
c_{n-m} c_{m-k}+c_{m-k} c_{k-n}+c_{k-n} c_{n-m}=\alpha,
$$

where $\alpha=0$ when $r$ is a trivial or triangular $r$-matrix and $\alpha=-\epsilon^{2}$ for $r$ quasitriangular (case (c)).

The wronskian $W$ of a plane configuration $w=(\phi, \psi)$ is defined by the obvious formula

$$
W[w]_{n}=\phi_{n} \psi_{n-1}-\psi_{n} \phi_{n-1} .
$$

The space $\mathcal{V} \subset \mathcal{W}$ of wave functions of discrete Schroedinger operators is defined by the constraint $W[w]=1$.

Proposition 3.2. We have

$$
\begin{aligned}
\left\{W_{n}, \phi_{m}\right\}=\left(a_{n-m}+a_{n-1-m}-\right. & \left.c_{n-m}\right) W_{n} \phi_{m} \\
& +\left(c_{n-m}-c_{n-m-1}\right)\left(\phi_{n} \phi_{n-1} \psi_{m}-\phi_{n} \psi_{n-1} \phi_{m}\right) .
\end{aligned}
$$

A similar formula holds for $\left\{W_{n}, \psi_{m}\right\}$.

Scaling invariants $\eta_{n}$ commute with the wronskian if and only if the second term in (34) is also proportional to $W_{n} \phi_{m}$; this condition implies that

$$
c_{n-m}-c_{n-m-1}=\epsilon\left(\delta_{n m}+\delta_{n, m+1}\right) .
$$

Without restricting the generality we may assume that $\epsilon=1$ and in that case we get

$$
\left\{W_{n}, \phi_{m}\right\}=\left(a_{n-m}+a_{n-1-m}-c_{n-m}+\delta_{n m}+\delta_{n, m+1}\right) W_{n} \phi_{m} .
$$

Fortunately, condition (35) is again satisfied by the sign function and hence the Poisson structure on the space of projective configurations remains basically the same as in the continuous case. Moreover, if $\eta$ is a projective curve, which defines a Schroedinger equation, we may fix a generic set of values $\left\{x_{1}, \ldots, x_{N}\right\}$ of the coordinate $x$ on the circle such that $\eta\left(x_{n}\right) \neq \eta\left(x_{n+1}\right)$; then $\left\{\eta\left(x_{n}\right)\right\}$ is a non-degenerate 
projective configuration which gives rise to a difference Schroedinger equation and the evaluation functionals $\eta \mapsto \eta\left(x_{n}\right)$ form a Poisson subalgebra in the big Poisson algebra (22). Explicitly we have

$$
\left\{\eta_{n}, \eta_{m}\right\}=\eta_{n}^{2}-\eta_{m}^{2}-\operatorname{sign}(n-m)\left(\eta_{n}-\eta_{m}\right)^{2}
$$

Note that it's of course not true that the solutions of this difference equation are the values of the wave functions for the continuous equation: indeed, the wronskian constraints are different in the two cases. It is noteworthy that nevertheless the conditions imposed by these constraints on the structure function $c$ are satisfied by the same standard function.

In order to compute the Poisson structure induced by (37) on the set of potentials let us start with the subfields of rational $N$ - and $B$-invariants in $\mathbb{C}(\eta)$; in complete analogy with the continuous case we have $\mathbb{C}(\eta)^{N}=\mathbb{C}(\theta)$, where $\theta_{m}:=\eta_{m+1}-\eta_{m}$, and $\mathbb{C}(\eta)^{B}=\mathbb{C}(\lambda)$, where

$$
\lambda_{m}:=\frac{\eta_{m+2}-\eta_{m+1}}{\eta_{m+1}-\eta_{m}}
$$

An easy computation yields

$$
\left\{\theta_{m}, \theta_{n}\right\}=-2 \operatorname{sign}(m-n) \theta_{m} \theta_{n}, \quad\left\{\lambda_{m}, \lambda_{n}\right\}=2\left(\delta_{m+1, n}-\delta_{m, n+1}\right) \lambda_{m} \lambda_{n} .
$$

A natural interpretation of the variables $\lambda_{n}$ is connected with the Miura transform for the discrete Schroedinger equation. Let us assume that the difference operator (29) is factorized,

$$
\tau^{2}+u \tau+1=(\tau+v)\left(\tau+v^{-1}\right) .
$$

The potentials $u, v$ are related by the difference Miura map,

$$
u_{n}=v_{n}+v_{n+1}^{-1} \text {. }
$$

We may assume without restricting the generality that $\psi$ is the solution of (28) which satisfies the first order equation $\left(\tau+v^{-1}\right) \psi=0$. Let $\phi$ be the second solution of this equation such that $W(\phi, \psi)=1$ and $\eta=\phi / \psi$; then

$$
\eta_{n+1}-\eta_{n}=\frac{1}{\psi_{n} \psi_{n+1}}
$$

Clearly, $v_{n}=-\psi_{n} / \psi_{n+1}$ and hence

$$
v_{n} v_{n+1}=\frac{\psi_{n}}{\psi_{n+2}}=\frac{\psi_{n+1} \psi_{n}}{\psi_{n+2} \psi_{n+1}}=\frac{\eta_{n+2}-\eta_{n+1}}{\eta_{n+1}-\eta_{n}}=\lambda_{n}
$$

Thus $\lambda_{n}$ is the product of two neighbouring potentials in the factorized Schroedinger operator (39). The potentials themselves again are not rational Galois invariants of $B$ and belong to a quadratic extension of $\mathbb{C}(\lambda)$. From (40), (41) we easily derive that

$$
s_{n}=u_{n} u_{n+1}=\frac{\left(1+\lambda_{n}\right)\left(1+\lambda_{n+1}\right)}{\lambda_{n+1}} .
$$


Proposition 3.3. We have

$$
\begin{aligned}
\left\{\lambda_{m}, \lambda_{n}\right\}= & \left(\delta_{m+1, n}-\delta_{m, n+1}\right) \lambda_{m} \lambda_{n}, \\
\left\{s_{m}, s_{n}\right\}= & \left(\delta_{m+1, n}-\delta_{m, n+1}\right)\left(s_{m}+s_{n}-s_{m} s_{n}\right) \\
& +s_{m} s_{n}\left(s_{m+1}^{-1} \delta_{m+2, n}-s_{n+1}^{-1} \delta_{m, n+2}\right) .
\end{aligned}
$$

Formula (43) implies the following Poisson bracket relations for the potentials:

Proposition 3.4. Let $\Phi_{n}=(-1)^{n} \operatorname{sign} n, n \neq 0, \Phi_{0}=0$. Then

$$
\left\{v_{n}, v_{m}\right\}=2 \Phi_{n-m} v_{n} v_{m} \text { and }\left\{u_{n}, u_{m}\right\}=2 \Phi_{n-m} u_{n} u_{m}+2\left(\delta_{m+1, n}-\delta_{m, n+1}\right) .
$$

Formula (44) coincides with the lattice Virasoro algebra introduced in [FRS], while (43) coincides with the Faddeev-Takhtajan version of the lattice Virasoro algebra. The non-locality of the Poisson bracket relations in (44) is due to the non-locality of the formula for potentials $v$ and $u$ in terms of $\eta$. The same structure constants $\Phi_{n-m}$ arise in [FRS] in the framework of the discrete Drinfeld-Sokolov theory, which provides for this formula a totally different (and more direct) explanation.

\section{ACKNOWLEDGEMENT}

The authors would like to thank L.D.Faddeev, V.Fock and V.Sokolov for useful discussions. The work of the second author was partially supported by the INTAS-OPEN grant 03-51-3350, the RFFI grant 05-01-00922 and the ANR program "GIMP" ANR-05-BLAN-0029-01. The first author is grateful to the Association Suisse-Russe for financing his visit to the Steklov Institute, with special thanks to J.-P.Periat and S.Yu.Sergueeva.

\section{REFERENCES}

[AMM] Alekseev, A., Malkin, A., Meinrenken, E. Lie group valued moment maps. J. Diff. Geom. 48 (1998), no. 3, 445-495.

[B1] Babelon, O. Extended conformal algebra and the Yang-Baxter equation, Phys. Lett. B 215 (1988), no. 3, 523-529.

[B2] Babelon, O. Exchange formula and lattice deformation of the Virasoro algebra, Phys. Lett. B 238 (1990), no. 2-4, 234-238.

[BB] Babelon, O., and Bernard, D. Dressing symmetries. Comm. Math. Phys., 149(2): 279-306, 1992.

[Bo] Boalch, P. Stokes matrices, Poisson Lie groups and Frobenius manifolds, Invent. math. 146, 479-506 (2001)

[CD] Calogero, F., Degasperis, A. Reduction technique for matrix nonlinear evolution equations solvable by the spectral transform. J. Math. Phys. 22 (1981), no. 1, 23-31.

[DS1] Drinfeld, V.G., Sokolov, V.V. Lie algebras and equations of Korteweg-de Vries type, Sov. Math. Dokl. 23 (1981) 457-462.

[DS2] Drinfeld, V.G., Sokolov, V.V. On equations related to the Korteweg-de Vries type, Sov. Math. Dokl. 32 (1985) 361-365.

[FT] Faddeev, L.D., Takhtajan, L.A. Liouville model on the lattice, Lect. Notes in Phys. 246 (1986), 166-179.

[FRS] Frenkel, E., Reshetikhin, N., Semenov-Tian-Shansky, M.A. Drinfeld-Sokolov reduction for difference operators and deformations of $W$-algebras. I: The case of Virasoro algebra, Commun. Math. Phys. 192 (1998) 605-629. 
[KN] Krichever, I.M., Novikov, S.P. Holomorphic bundles over algebraic curves, and nonlinear equations. Russian Math. Surveys 35 (1980), no. 6, 53-80.

[OT] Ovsienko, V., Tabachnikov, S. Projective differential geometry old and new: from Schwarzian derivative to cohomology of diffeomorphism group. Cambridge Tracts in Mathematics, 165. Cambridge University Press, Cambridge, 2005.

[S1] Semenov-Tian-Shansky, M.A. Dressing action transformations and Poisson-Lie group actions, Publ. RIMS. 21 (1985), 1237-1260.

[S2] Semenov-Tian-Shansky, M.A. Monodromy map and classical r-matrices. Zapiski nauchn. semin. POMI, v. 200, 156-166, 1992 (Russian); Preprint hep-th 9402022, 1994.

[STSS] Semenov-Tian-Shansky, M.A., Sevostyanov, A.V. Drinfeld-Sokolov reduction for difference operators and deformations of $W$-algebras. II: The general semisimple case, Commun. Math. Phys. 192 (1998) 631-647.

[SS] Svinolupov, S.I., Sokolov, V.V. Evolution equations with nontrivial consevation laws, Funct. Anal. Appl. 16 (1983), 317-319.

[V] Volkov, A.Yu. Miura transformation on a lattice, Theor. Math. Phys. 74 (1988), 96-99.

[W1] Wilson, G. On the quasi-Hamiltonian formalism of the KdV equation, Physics Letters A 132 (1988), 445-450.

[W2] Wilson, G. On antiplectic pairs in the Hamiltonian formalism of evolution equations, Quart. J. Math. Oxford Ser.(2) 42 (1991), 227-256.

[W3] Wilson, G. On the antiplectic pair connected with the Adler-Gel'fand-Dikii bracket, Nonlinearity 5 (1992), 109-131.

Mathematics Department, University of Loughborough, UK

E-mail address: ian.marshall@math.unige.ch

Institut Mathématique de Bourgogne, Dijon, France and Steklov Mathematical Institute, St. Petersburg, Russia

E-mail address: semenov@u-bourgogne.fr 